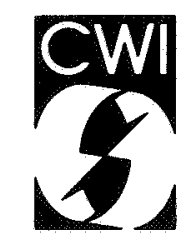

Centrum voor Wiskunde en Informatica Centre for Mathematics and Computer Science

W.H. Hundsdorfer, J. Schneid

On the equivalence of BS-stability and B-consistency

- Department of Numerical Mathematics Report NM-R8815 November


The Centre for Mathematics and Computer Science is a research institute of the Stichting Mathematisch Centrum, which was founded on February 11, 1946, as a nonprofit institution aiming at the promotion of mathematics, computer science, and their applications. It is sponsored by the Dutch Government through the Netherlands Organization for the Advancement of Pure Research (Z.W.O.). 


\title{
On the Equivalence of BS-Stability and B-Consistency
}

\author{
W.H. Hundsdorfer \\ Centre for Mathematics and Computer Science \\ P.O. Box 4079, 1009 AB Amsterdam, The Netherlands \\ J. Schneid \\ Institut für Angewandte und Numerische Mathematik \\ Technische Universität Wien \\ Wiedner Hauptstrasse 8-10, A-1040 Vienna, Austria
}

\begin{abstract}
Among several stability and consistency concepts for Runge-Kutta methods applied to stiff initial value problems, BS-stability and B-consistency turn out to be equivalent for initial value problems with a onesided Lipschitz constant $m \geqslant 0$. In addition to this result, it is shown that the same holds for their internal counterparts.
\end{abstract}

1980 Mathematics Subject Classification: 65L05.

Key Words \& Phrases: stiff initial value problems, implicit Runge-Kutta methods, B-convergence.

Note: This paper was written while J. Schneid was visiting the Centre for Mathematics and Computer

Science with an Erwin-Schrödinger stipend from the Fonds zur Förderung der wissenschaftlichen Forschung.

\section{INTRODUCTION}

In the last decade several stability concepts have been introduced for assessing convergence of Runge-Kutta methods applied to stiff nonlinear differential equations $y^{\prime}=f(t, y)$ with $f$ satisfying a one-sided Lipschitz condition with constant $m$. Among these, $B$-stability and the equivalent criterium of algebraic stability are well-known (see [2], [3], [6]); they guarantee stability w.r.t. perturbations of the initial value for $m \leqslant 0$. Moreover, they enable establishing $B$-convergence (i.e. global error bounds independent of the stiffness, see [7]) for $m<0$ ([5], [11]); for $m \geqslant 0$, however, $B$-stability is not sufficient to have such convergence property (as was shown by means of a counterexample in [12]). For a nonnegative $m B$-convergence can be proved if, in addition, $B S$ - and $B S I$ - stability are assumed, ensuring stability per step w.r.t. perturbations of the internal stages of the Runge-Kutta method ([8], [9], [10]).

In this paper it will be shown that for any $m \geqslant 0 B S$-stability is equivalent to $B$-consistency (i.e. local error bounds independent of the stiffness, see [7]), revealing the necessity of $B S$-stability for stiffness independent error bounds; for $B S I$-stability a similar equivalence will be derived.

Report NM-R8815

Centre for Mathematics and Computer Science

P.O. Box 4079, 1009 AB Amsterdam, The Netherlands 


\section{Preliminaries}

Consider the numerical solution of a stiff initial value problem

$$
\begin{aligned}
& y^{\prime}=f(t, y), f:[0, T] \times \mathbb{R}^{n} \rightarrow \mathbb{R}^{n}, T>0, \\
& y(0)=y_{0}, y_{0} \in \mathbb{R}^{n}
\end{aligned}
$$

by an $s$-stage Runge-Kutta method

$$
\begin{aligned}
& Y_{i}^{k}=\eta_{k-1}+h \sum_{j=1}^{s} a_{i j} f\left(t_{k-1}+c_{j} h, Y_{j}^{k}\right), 1 \leqslant i \leqslant s, \\
& \eta_{k}=\eta_{k-1}+h \sum_{j=1}^{s} b_{j} f\left(t_{k-1}+c_{j} h, Y_{j}^{k}\right)
\end{aligned}
$$

with $\eta_{0}=y_{0}$ and $\eta_{k} \approx y\left(t_{k}\right)$ for $k=1,2, \ldots$ and $t_{k}=k h \leqslant T$. It will be assumed, for convenience, that the nodes are such that $0 \leqslant c_{1} \leqslant \cdots \leqslant c_{s} \leqslant 1$. Using the abbreviations

$$
Y^{k}=\left[\begin{array}{c}
Y_{1}^{k} \\
\vdots \\
Y_{s}^{k}
\end{array}\right], \quad F(t, U)=\left[\begin{array}{c}
f\left(t+c_{1} h, U_{1}\right) \\
\vdots \\
f\left(t+c_{s} h, U_{s}\right)
\end{array}\right]
$$

for $U=\left(U_{i}\right), U_{i} \in \mathbb{R}^{n}$, and $e=(1, \ldots, 1)^{T} \in \mathbb{R}^{s}$, system (2.2) will be written from now on as

$$
\begin{aligned}
& Y^{k}=\mathbf{e} \eta_{k-1}+h \mathbf{A} F\left(t_{k-1}, Y^{k}\right) \\
& \eta_{k}=\eta_{k-1}+h \mathbf{b}^{T} F\left(t_{k-1}, Y^{k}\right) .
\end{aligned}
$$

Here, boldface letters indicate Kronecker products with $I_{n}$ to make $A=\left(a_{i j}\right), b=\left(b_{i}\right)$ and $e$ of appropriate dimension. Further, $\langle v, w\rangle=v^{T} w$ denotes the Euclidean inner product on $\mathbb{R}^{l}, l \in \mathbb{N}$, with $\|\cdot\|$ as the corresponding norm.

The righthand side $f$ of (2.1a) is assumed to be differentiable w.r.t. $y$ and to satisfy a one-sided Lipschitz condition (with a one-sided Lipschitz constant $m$ )

$$
<f\left(t, y_{1}\right)-f\left(t, y_{2}\right), y_{1}-y_{2}>\leqslant m\left\|y_{1}-y_{2}\right\|^{2}
$$

for all $t \in[0, T]$ and $y_{1}, y_{2} \in \mathbb{R}^{n}$. The set of all functions $f$ in (2.1a) satisfying (2.4) for a given $m \in \mathbb{R}$ will be denoted by $\mathscr{F}_{m}$.

Local stability and local errors of a Runge-Kutta method can be studied by considering the difference between an unperturbed step

$$
Y=\mathbf{e} \eta_{0}+h \mathbf{A} F\left(t_{0}, Y\right)
$$




$$
\eta_{1}=\eta_{0}+h \mathbf{b}^{T} F\left(t_{0}, Y\right)
$$

and a perturbed one,

$$
\begin{aligned}
& \tilde{Y}=\mathbf{e} \eta_{0}+h \mathbf{A} F\left(t_{0}, \tilde{Y}\right)+\Delta \\
& \tilde{\eta}_{1}=\eta_{0}+h \mathbf{b}^{T} F\left(t_{0}, \tilde{Y}\right)+\delta,
\end{aligned}
$$

with perturbations $\Delta \in \mathbb{R}^{n s}$ and $\delta \in \mathbb{R}^{n}$. Introducing $V=\tilde{Y}-Y, v=\tilde{\eta}_{1}-\eta_{1} \quad$ and $W=h F\left(t_{0}, \tilde{Y}\right)-h F\left(t_{0}, Y\right)$, the difference between (2.6) and (2.5) can be written as

$$
\begin{aligned}
& V=\mathbf{A} W+\Delta \\
& v=\mathbf{b}^{T} W+\delta .
\end{aligned}
$$

Now, the Runge-Kutta method is called BSI-stable on $\mathscr{F}_{m}$ if there are constants $\alpha_{0}, D_{0}>0$ such that

$$
\|V\| \leqslant D_{0}\|\Delta\|
$$

for every $h>0$ with $h m \leqslant \alpha_{0}$, and for all $\Delta \in \mathbb{R}^{n s}$ and $f \in \mathscr{F}_{m}$. The Runge-Kutta method is called $B S$ stable on $\mathscr{F}_{m}$ if there are constants $\alpha_{1}, D_{1}>0$ such that

$$
\|v\| \leqslant D_{1}(\|\Delta\|+\|\delta\|)
$$

for every $h>0$ with $h m \leqslant \alpha_{1}$, and for all $\Delta \in \mathbb{R}^{n s}, \delta \in \mathbb{R}^{n}$ and $f \in \mathscr{F}_{m}$.

In order to study local errors let $y(t)$ be a solution of (2.1a) with $f \in \mathscr{F}_{m}$; further, let $M_{j}=\max \left\{\left\|y^{(j)}(t)\right\| 10 \leqslant t \leqslant T\right\}$. Then the method is called BI-consistent on $\mathscr{F}_{m}$ if there are constants $\beta_{0}>0, q_{0}>0$ (depending only on the method) and $C_{0}>0$ (depending on some of the $M_{j}$ 's but independent of the stiffness of the problem) such that

$$
\left\|y\left(t_{0}+c_{i} h\right)-Y_{i}\right\| \leqslant C_{0} h^{q_{0}}, \quad 1 \leqslant i \leqslant s,
$$

for every $h>0$ with $h m \leqslant \beta_{0}$ and for every solution $y$ of (2.1a) with $f \in \mathscr{F}_{m}$. Finally, the method is called $B$-consistent on $\mathscr{F}_{m}$ if there are constants $\beta_{1}>0, q_{1}>0$ and $C_{1}>0$ such that

$$
\left\|y\left(t_{1}\right)-\eta_{1}\right\| \leqslant C_{1} h^{q_{1}}
$$

for every $h>0$ with $h m \leqslant \beta_{1}$ and for every solution $y$ of (2.1a) with $f \in \mathscr{F}_{m}$; again, for $\beta_{1}, q_{1}$ and $C_{1}$ the same holds as said above.

Both $B(I)$-consistency and $B S(I)$-stability are crucial for assessing $B$-convergence results for 
Runge-Kutta methods on $\mathscr{T}_{m}, m \geqslant 0$ (see e.g. [9]).

There has been no specification yet about the dimension $n$. For convenience, $n$ will be considered as a fixed but arbitrary integer, which means that all bounds $D_{i}, C_{i}$ are allowed to depend on $n$. Results uniformly in $n$, similar to those that will be derived for fixed $n$, can be obtained in the same way by considering all the spaces $\mathbb{R}^{n}$ as subspaces of the Hilbert space $l_{2}$. Furthermore, also $T$ is considered as a fixed number; its value is irrelevant since $B S(I)$-stability and $B(I)$-consistency are all local properties related to one step of the Runge-Kutta scheme.

It is pointed out, finally, that throughout this paper it is tacitly assumed that the system of algebraic equations defining the internal vectors $Y_{i}$ has a unique solution, although the question of solvability is somewhat related to the concept of BSI-stability.

\section{EQUIVALENCE OF $B S(I)$-STABILITY AND $B(I)$-CONSISTENCY}

The main theorem of this paper is stated here without proof; it will be given in the next section.

THEOREM 3.1. Suppose $e_{1}^{T} A \neq 0, c_{i} \neq c_{j}$ whenever $i \neq j$, and let $m \geqslant 0$. Then the Runge-Kutta method is $B S$-stable on $\mathscr{F}_{m}$ if and only if it is B-consistent on $\mathscr{F}_{m}$. Likewise, the method is BSI-stable on $\mathscr{F}_{m}$ if and only if it is BI-consistent on $\mathscr{F}_{m}$.

The condition $e_{1}^{T} A \neq 0$ cannot be omitted as the trapezoidal rule would then pose a counterexample to the theorem: for any $m \in \mathbb{R}$ it is $B$ - and $B I$-consistent on $\mathscr{F}_{m}$ but neither $B S$ - nor $B S I$-stable on $\mathscr{F}_{m}$. Note further that the theorem is not valid for $m<0$ since the Lobatto IIIC scheme with three stages is known to be $B$ - and $B I$-consistent on $\mathscr{F}_{m}$ for $m<0$ (see [5], [11]), but neither BSI-stable (see [4]) nor $B S$-stable on $\mathscr{F}_{m}$ for any $m \in \mathbb{R}$, as can easily be shown.

\section{Proofs}

In a first step algebraic criteria for $B S I$ - and $B S$-stability will be given for nonconfluent methods; nonconfluency means that $c_{i} \neq c_{j}$ whenever $i \neq j$. The logarithmic norm of a matrix $Z$ will be denoted by $\mu(Z)$ and $\mathscr{D}_{m}$ will stand for the collection of matrices $\mathbf{Z}=\operatorname{blockdiag}\left(Z_{1}, \ldots, Z_{s}\right) \in \mathbb{R}^{n s \times n s}$ with $Z_{i} \in \mathbb{R}^{n \times n}$ and $\mu\left(Z_{i}\right) \leqslant m$. The identity matrix of dimension $n s$ will be denoted by $\mathbf{I}$.

Lemma 4.1. A nonconfluent Runge-Kutta method is BSI-stable on $\mathscr{F}_{m}$ if and only if there are constants $\alpha_{0}, D_{0}>0$ such that

$$
\left\|(\mathrm{I}-h \mathrm{AZ})^{-1}\right\| \leqslant D_{0}
$$


for every $h>0$ with $h m \leqslant \alpha_{0}$ and $\mathbf{Z} \in \mathscr{D}_{m}$. The method is BS-stable on $\mathscr{F}_{m}$ if and only if there are constants $\alpha_{1}, D_{1}>0$ such that

$$
\left\|h \mathbf{b}^{T} \mathbf{Z}(\mathbf{I}-h \mathbf{A Z})^{-1}\right\| \leqslant D_{1}
$$

for every $h>0$ with $h m \leqslant \alpha_{1}$ and $\mathbb{Z} \in \mathscr{D}_{m}$.

Proof. The difference (2.7) between an unperturbed and a perturbed step can be written as

$$
\begin{aligned}
& V=h \mathbf{A Z Z} V+\Delta \\
& v=h \mathbf{b}^{T} \mathbf{Z} V+\delta
\end{aligned}
$$

where $\mathbf{Z}$ is the blockdiagonal matrix with blocks $Z_{i}=\int_{0}^{1} f_{y}\left(t_{0}+c_{i} h, Y_{i}+\theta\left(\tilde{Y}_{i}-Y_{i}\right)\right) d \theta$, and - due to (2.4) $-\mathbb{Z} \in \mathscr{D}_{m}$. So (4.1) takes the form

$$
\begin{aligned}
& V=(\mathbb{I}-h \mathbf{A Z Z})^{-1} \Delta \\
& v=h \mathbf{b}^{T} \mathbf{Z}(\mathbb{I}-h \mathbf{A Z Z})^{-1} \Delta+\delta
\end{aligned}
$$

and both $B S I$ - and $B S$-stability directly follow from the upper bounds given in the lemma.

The necessity of these bounds can easily be seen by considering out of $\mathscr{F}_{m}$ the class of problems

$$
y^{\prime}=\Lambda(t) y, \quad \Lambda(t) \in \mathbb{R}^{n \times n}, \quad \mu(\Lambda(t)) \leqslant m, \quad t \in[0, T],
$$

and taking $\delta=0$. Then $Z_{i}=\Lambda\left(t_{0}+c_{i} h\right)$ can be chosen arbitrary and independent of each other as $c_{i} \neq c_{j}$ whenever $i \neq j$ was assumed.

In order to relate $B S(I)$-stability and $B(I)$-consistency, particular perturbations will be considered. For any differentiable function $g:[0, T] \rightarrow \mathbb{R}^{n}$ define $\Delta(g) \in \mathbb{R}^{n s}$ and $\delta(g) \in \mathbb{R}^{n}$ by

$$
\begin{aligned}
& \Delta_{i}(g)=g\left(t_{0}+c_{i} h\right)-g\left(t_{0}\right)-h \sum_{j=1}^{s} a_{i j} g^{\prime}\left(t_{0}+c_{j} h\right), \quad 1 \leqslant i \leqslant s, \\
& \delta(g)=g\left(t_{1}\right)-g\left(t_{0}\right)-h \sum_{i=1}^{s} b_{i} g^{\prime}\left(t_{0}+c_{i} h\right) .
\end{aligned}
$$

LEMMA 4.2. Suppose the Runge-Kutta method is nonconfluent and $e_{1}^{T} A \neq 0$. Then for any $\Delta \in \mathbb{R}^{n s}$ and $h>0$ there is a differentiable function $g$ such that $\Delta(g)=\Delta$.

Proof. Consider arbitrary $u_{0}, u_{i}, w_{i} \in \mathbb{R}^{n}, 1 \leqslant i \leqslant s$, with the restriction that $u_{0}=u_{1}$ in the case $c_{1}=0$. Since the Runge-Kutta method was assumed to be nonconfluent the function $g$ can be chosen as a 
polynomial on $[0, T]$ with coefficients in $\mathbb{R}^{n}$ such that $g\left(t_{0}\right)=u_{0}, g\left(t_{0}+c_{i} h\right)=u_{i}$, and $g^{\prime}\left(t_{0}+c_{i} h\right)=w_{i}$ for $1 \leqslant i \leqslant s$. With $u=\left(u_{i}\right)$ and $w=\left(w_{i}\right) \in \mathbb{R}^{n s}$ the equation $\Delta(g)=\Delta$ then holds if and only if $u-\mathbf{e} u_{0}-h \mathrm{~A} w=\Delta$ holds. For any given $\Delta$ and $h$, though, such vectors $u_{0}, u$ and $w$ exist because of the assumption that $e_{1}^{T} A \neq 0$ for the case $c_{1}=0$.

With the help of the above two lemmas Theorem 3.1 can now be proved.

Consider first (2.6) with $\Delta=\Delta(y)$ and $\delta=\delta(y)$ where $y$ denotes the solution of (2.1); then $\tilde{Y}_{i}=y\left(t_{0}+c_{i} h\right), \tilde{\eta}_{1}=y\left(t_{1}\right)$, and thus $B S(I)$-stability on $\mathscr{F}_{m}$ implies $B(I)$-consistency on $\mathscr{F}_{m}$ for arbitrary $m \in \mathbb{R}$ (see also [9]).

Assume now a nonconfluent Runge-Kutta method with $e_{1}^{T} A \neq 0$ to be $B I$-consistent on $\mathscr{F}_{m}, m \geqslant 0$. Choose out of $\mathscr{F}_{m}$ the class of problems

$$
\begin{aligned}
& y^{\prime}=\Lambda(t)(y-g(t))+g^{\prime}(t) \\
& y(0)=g(0)
\end{aligned}
$$

with $\Lambda(t) \in \mathbb{R}^{n \times n}$ such that $\mu(\Lambda(t)) \leqslant m$ for all $t \in[0, T]$ and with arbitrary $g:[0, T] \rightarrow \mathbb{R}^{n}$ as the solution $y(t)$. The $B I$-consistency inequality (2.10) together with (4.2) implies

$$
\left\|(\mathbf{I}-h \mathbf{A Z})^{-1} \Delta(g)\right\| \leqslant \sqrt{s} C_{0} h^{q_{0}}
$$

for every $h>0$ with $h m \leqslant \beta_{0}$, where $\mathbb{Z}=\operatorname{blockdiag}\left(Z_{1}, \ldots, Z_{s}\right)$ and $Z_{i}=\Lambda\left(t_{0}+c_{i} h\right)$; the constant $C_{0}$ depends on some bounds $M_{j}$ for $\left\|g^{(j)}(t)\right\|, t \in[0, T]$, but is independent of the stiffness of the problem. From the assumption that $c_{i} \neq c_{j}$ whenever $i \neq j$ it follows that $\mathbb{Z}$ can be any matrix in $\mathscr{D}_{m}$ by choosing $\Lambda(t)$ appropriately. Lemma 4.2 now implies the existence of a positive function $\phi(h, \Delta)$ such that

$$
\left\|(\mathbf{I}-h \mathbf{A Z})^{-1} \Delta\right\| \leqslant \phi(h, \Delta)
$$

for any $\Delta \in \mathbb{R}^{n s}, \mathbb{Z} \in \mathscr{D}_{m}$ and $0<h \leqslant H=\min \left\{T, \beta_{0} / m\right\}(H=T$ if $m=0)$. Note that $\mathbf{Z} \in \mathscr{D}_{m}$ if and only if $h \mathbf{Z} \in \mathscr{D}_{h m}$ and that $\mathscr{D}_{h m} \subset \mathscr{D}_{\mathrm{Hm}}$ as $m \geqslant 0$; consequently,

$$
\sup _{\mathbf{Z} \in \omega_{m}}\left\|(I-h \mathbf{A Z})^{-1} \Delta\right\| \leqslant \sup _{\mathbf{Z} \in \omega_{m}}\left\|(I-H \mathbf{A Z})^{-1} \Delta\right\|
$$

Taking $\psi(\Delta)=\phi(H, \Delta)$ gives

$$
\left\|(\mathbf{I}-h \mathbf{A Z})^{-1} \Delta\right\| \leqslant \psi(\Delta)
$$

for any $\Delta \in \mathbb{R}^{n s}, \mathbf{Z} \in \mathscr{D}_{m}$ and $0<h \leqslant H$. From the principle of uniform boundedness (see e.g. [1]) it now follows that $(\mathbb{I}-h \mathbf{A Z})^{-1}$ is uniformly bounded for $\mathbf{Z} \in \mathscr{D}_{m}$ and $0<h \leqslant H$. By the characterization of 
Lemma 4.1 the method is thus $B S I$-stable on $\mathscr{F}_{m}, m \geqslant 0$.

Finally, assuming $B$-consistency on $\mathscr{F}_{m}, m \geqslant 0$, and choosing again the class of problems (4.4), the $B$-consistency inequality (2.11) together with (4.2) implies

$$
\left\|h \mathbf{b}^{T} \mathbf{Z}(\mathbf{I}-h \mathbf{A Z Z})^{-1} \Delta(g)\right\| \leqslant C_{1} h^{q_{1}}+\|\delta(g)\|
$$

for every $h>0$ with $h m \leqslant \beta_{1}$ and $\mathbf{Z} \in \mathscr{D}_{m}$. Expanding $\delta(g)$ into a Taylor series yields

$$
\|\delta(g)\| \leqslant C M_{q} h^{q}
$$

for some $C>0, q \in \mathbb{N}$. Again, by using the principle of uniform boundedness and the characterization of Lemma 4.1 BS-stability can be established.

\section{REFERENCES}

[1] G. Bachman, L. Narici, Functional Analysis, New York - London: Academic Press 1966.

[2] K. BurRage, J.C. BUTCher, Stability Criteria for Implicit Runge-Kutta Methods, SIAM J. Num. Anal. 16, 46-57 (1979).

[3] M. Crouzeix, Sur la B-stabilité des méthodes de Runge-Kutta, Numer. Math. 32, 75-82 (1979).

[4] K. DeKKER, E. HAIRER, A Necessary Condition for BSI-stability, BIT 25, 285-288 (1985).

[5] K. DekKer, J.F.B.M. KraAiJeVAnger, J. SchneID, On the Relation between Algebraic Stability and B-convergence for Runge-Kutta Methods, Submitted to Numer. Math.

[6] K. DekKER, J.G. VERWER, Stability of Runge-Kutta Methods for Stiff Nonlinear Differential Equations, Amsterdam-New York-Oxford: North Holland 1984.

[7] R. Frank, J. Schneid, C.W. Ueberhuber, The Concept of B-convergence, SIAM J. Num. Anal. $18,753-780(1981)$.

[8] R. Frank, J. Schneid, C.W. Ueberhuber, Stability Properties of Implicit Runge-Kutta Methods, SIAM J. Num. Anal. 22, 497-515 (1985).

[9] R. Frank, J. SchneID, C.W. UeberhUBER, Order Results for Implicit Runge-Kutta Methods Applied to Stiff Systems, SIAM J. Num. Anal. 22, 515-534 (1985).

[10] W.H. HUNDSDORFER, Convergence of Runge-Kutta Methods on Classes of Stiff Initial Value Problems, In: B. Karasözen (ed.), Proceedings Int. Symp. on Num. Anal., METU, Ankara (1987).

[11] J. SCHNEID, Characterization of B-convergent Runge-Kutta Methods for Strictly Dissipative Initial Value Problems, Submitted to Computing.

[12] M.N. SpIJKER, The Relevance of Algebraic Stability in Implicit Runge-Kutta Methods, In: K. Strehmel, (ed.), Num. Treatment of Diff. Equations, pp. 150-164, Leipzig: Teubner 1986. 
